\title{
Model-Free Adaptive Predictive Control for Networked Control Systems with Dual-Channel Packet Loss
}

\author{
Shuo Zhen ${ }^{1, a}$ Wei Gao ${ }^{1, b}$ \\ ${ }^{12}$ Beijing Jiaotong University, School of Electronic and Information Engineering, Beijing, China, \\ 100044 \\ aemail,
}

\begin{abstract}
Keywords: Networked Control Systems, Model-Free Adaptive Predictive Control, Data-Driven
\end{abstract} Control, Random Packet Loss

\begin{abstract}
In order to reduce the impact of network communication on networked control system (NCS), a networked model-free adaptive predictive control scheme is proposed for networked control systems with random packet loss in the forward and feedback channels. The data model based on the I/O data dynamic linearization is used as the predictive model. It is a data driven predictive control method and it is independent of the mathematical model for the controlled object. To suppress the system oscillation caused by packet loss and the frequent change of the expected signal, an improved MFAPC method was proposed. Finally, the effectiveness of the improved MFAPC method for packet loss was verified by simulation.
\end{abstract}

\section{Introduction}

Networked control system (NCS) is a distributed feedback control system which transmits data through communication network ${ }^{[1]}$. The introduction of network makes NCS easy to realize remote control and resource sharing. However, the introduction of the network has also brought new challenges. When the sensor, the controller and the actuator transmit data through the network, data collision, multi-packet transmission and multi-path transmission may appear, which may cause packet loss and network-induced delay problem inevitably.

Packet loss and network-induced delay are two important factors that can cause NCS's stability degradation and even instability. Therefore, reducing the impact of data packet loss and network-induced delay on system performance has become a hotspot. In practice, industrial process control system is slow, packet loss is a more important issue compared to network-induced delay ${ }^{[2]}$. The way to deal with packet loss can be divided into two methods: model based method ${ }^{[3-6]}$ and data-driven method ${ }^{[7-9]}$.

In this paper, we propose a networked model-free adaptive predictive control (MFAPC) scheme for NCS with random packet loss in the forward channel and the feedback channel. MFAPC does not rely on the mathematical model of the plant, which is a data-driven predictive control method. In order to suppress the system oscillation caused by the frequent change of the desired signal and the packet loss, an improved MFAPC method is proposed. Finally, the effectiveness of the proposed scheme is verified by simulation.

\section{Problem Description}

Consider a discrete-time SISO nonlinear system:

$$
y(k+1)=f\left(y(k), y(k-1), \ldots, y\left(k-n_{y}\right), u(k), u(k-1), \ldots, u\left(k-n_{u}\right)\right)
$$

where, $u(k) \in \mathbf{R}, y(k) \in \mathbf{R}$ are the input and output respectively. $f(\ldots): \mathbf{R}^{n_{u}+n_{y}+2} \rightarrow \mathbf{R}$ is a unknown nonlinear function, $n_{y}, n_{u}$ are unknown positive integers.

The structure of the NCS is shown in Fig.1. The NCS consists of sensor, networked controller, actuator, compensator and network. The sensor, actuator and compensator are time-driven, and the controller is event-driven. 


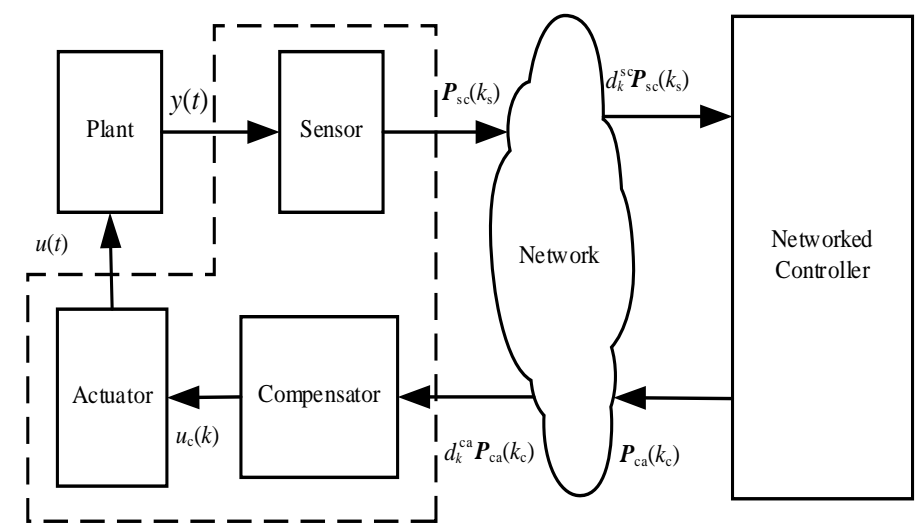

Fig.1 The Structure of NCS

\section{The Improved Model-Free Adaptive Predictive Control Method}

The MFAPC ${ }^{[10]}$ is a data-driven predictive control method whose prediction model is a kind of data model based on the dynamic linearization method. And the predictive model is independent of the controlled system's mathematical model.

The Data Model Based on Dynamic Linearization. The discrete-time SISO nonlinear system (2.1) can be transformed into a dynamic linearized model under certain conditions ${ }^{[13]}$

$$
\hat{y}(k+1)=y(k)+\varphi_{\mathrm{c}}(k) \Delta u(k)
$$

where $\varphi_{\mathrm{c}}(k) \in \mathbf{R}$ is the pseudo partial derivative (PPD) of the system.

Then, the following $N$-step forward prediction model can be given

$$
\hat{\boldsymbol{Y}}_{N}(k+1 \mid k)=\boldsymbol{C}(k) y(k)+\hat{\boldsymbol{A}}(k) \Delta \boldsymbol{U}_{N}(k)
$$

where $\hat{\boldsymbol{Y}}_{N}(k+1 \mid k)=[\hat{y}(k+1 \mid k), \ldots, \hat{y}(k+N \mid k)]^{\mathrm{T}}, \boldsymbol{C}(k)=[1,1, \ldots, 1]_{1 \times N}^{\mathrm{T}}, \boldsymbol{e}(k)=[e(k), e(k+1), \cdots, e(k+N)]^{\mathrm{T}}$,

$$
\begin{gathered}
\Delta \boldsymbol{U}_{N}(k)=[\Delta u(k \mid k), \ldots, \Delta u(k+N-1 \mid k)]^{\mathrm{T}}, \\
\hat{\boldsymbol{A}}(k)=\left[\begin{array}{cccc}
\hat{\varphi}_{\mathrm{c}}(k \mid k) & 0 & \ldots & 0 \\
\hat{\varphi}_{\mathrm{c}}(k \mid k) & \hat{\varphi}_{\mathrm{c}}(k+1 \mid k) & \ldots & 0 \\
\vdots & \vdots & \ddots & \vdots \\
\hat{\varphi}_{\mathrm{c}}(k \mid k) & \ldots & & 0 \\
\vdots & & & \vdots \\
\hat{\varphi}_{\mathrm{c}}(k \mid k) & \hat{\varphi}_{\mathrm{c}}(k+1 \mid k) & \cdots & \hat{\varphi}_{\mathrm{c}}(k+N-1 \mid k)
\end{array}\right]_{N \times N} .
\end{gathered}
$$

Rolling Optimization. For the predictive model (3.2), the optimal control value can be obtained under the constraint of the quadratic criterion

$$
J=\mid \boldsymbol{Y}_{N}^{*}(k+1)-\hat{\boldsymbol{Y}}_{N}(k+1 \mid k)\left\|^{2}+\lambda\right\| \Delta \boldsymbol{U}_{N_{u}}(k) \|^{2}
$$

where $\boldsymbol{Y}_{N}^{*}(k+1)=\left[y^{*}(k+1), \ldots, y^{*}(k+N)\right]^{\mathrm{T}}, \lambda>0$ is the weighting factor, $y^{*}(k+i)$ is the desired output at time $k+i(i=1, \ldots, N)$.

Substituting (3.5) into (3.4), let $\partial J / \partial \boldsymbol{U}_{N_{u}}(k)=0$, the MFAPC control increment can be obtained

$$
\Delta \boldsymbol{U}_{N_{u}}(k)=\left[\hat{\boldsymbol{A}}_{1}^{\mathrm{T}}(k) \hat{\boldsymbol{A}}_{1}(k)+\lambda \boldsymbol{I}\right]^{-1} \hat{\boldsymbol{A}}_{1}^{\mathrm{T}}(k)\left[\boldsymbol{Y}_{N}^{*}(k+1)-\boldsymbol{C}(k) y(k)\right]
$$

It can be seen that (3.5) only has a similar integral structure. Therefore, when the desired signal set value is changed frequently, the system is prone to oscillate. To suppress turbulence in the system, an improved quadratic criterion is given as follows

$$
J=k_{\mathrm{I}}\left\|\boldsymbol{Y}_{N}^{*}(k+1)-\hat{\boldsymbol{Y}}_{N}(k+1 \mid k)\right\|^{2}+k_{\mathrm{P}}\left\|\Delta \boldsymbol{Y}_{N}^{*}(k+1)-\Delta \hat{\boldsymbol{Y}}_{N}(k+1 \mid k)\right\|^{2}+\lambda\left\|\Delta \boldsymbol{U}_{N_{u}}(k)\right\|^{2}
$$

Substituting (3.5) into (3.6), and Let $\partial J / \partial \boldsymbol{U}_{N_{u}}(k)=0$, we get the improved control increment by

$$
\Delta \boldsymbol{U}_{N_{u}}(k)=\left[\lambda \boldsymbol{I}+\left(k_{\mathrm{i}}+k_{\mathrm{p}}\right) \boldsymbol{A}_{1}^{T} \boldsymbol{A}_{1}\right]^{-1} \boldsymbol{A}_{1}^{T}(k)\left[k_{\mathrm{p}}\left(\boldsymbol{Y}_{N}^{*}(k+1)-\boldsymbol{Y}_{N}^{*}(k)+\boldsymbol{Y}_{N}(k)-\boldsymbol{C}(k) y(k)\right)+k_{\mathrm{i}}\left(\boldsymbol{Y}_{N}^{*}(k+1)-\boldsymbol{E} y(k)\right)\right]
$$

Finally, the control value is obtained by 
where $\boldsymbol{g}=[1,0, \ldots, 0]^{\mathrm{T}}$.

$$
u(k)=u(k-1)+\boldsymbol{g}^{\mathrm{T}} \Delta \boldsymbol{U}_{N_{u}}(k)
$$

Parameter Online Estimation. We use an improved projection method ${ }^{[13]}$ to estimate $\varphi_{\mathrm{c}}(k)$, and the other parameters $\hat{\varphi}_{\mathrm{c}}(k+i \mid k)$ can be given by auto regression model.

$$
\hat{\varphi}_{\mathrm{c}}(k)=\hat{\varphi}_{\mathrm{c}}(k-1)+\frac{\eta \Delta u(k-1)}{\mu+\Delta u(k-1)^{2}}\left[\Delta y(k)-\hat{\varphi}_{\mathrm{c}}(k-1) \Delta u(k-1)\right]
$$

where $\mu>0$ is the weighting factor, $0<\eta \leq 1$ is the step factor.

\section{NCS Control Scheme Based on MFAPC}

In order to compensate the lost packet data, the networked MFAPC control schema was proposed as Fig.1 shows. Now give the detail designs as follow.

Sensor Node. At every sampling time, sensor node sends $\boldsymbol{P}_{\mathrm{sc}}(k)$ packet to controller node.

$$
\boldsymbol{P}_{\mathrm{sc}}(k)=\left[\begin{array}{lll}
\boldsymbol{Y}^{*} & \left(k_{\mathrm{s}}+1\right)^{\mathrm{T}} \quad y\left(k_{\mathrm{s}}\right) & k_{\mathrm{s}}
\end{array}\right]^{\mathrm{T}}
$$

where $\boldsymbol{Y}^{*}\left(k_{\mathrm{s}}+1\right)=\left[y^{*}\left(k_{\mathrm{s}}+1\right) y^{*}\left(k_{\mathrm{s}}+2\right) \ldots y^{*}\left(k_{\mathrm{s}}+N\right)\right]^{\mathrm{T}}, y^{*}(\ldots)$ is desired signal, $y(k)$ is system's output, $k_{\mathrm{s}}$ is sensor timestamp.

Controller Node. The networked predictive controller adopts MFAPC method. $\hat{A}_{1}(k)$ is calculated by (3.9), (3.8) yield the following control increment sequence

$$
\Delta \boldsymbol{U}_{k_{\mathrm{s}}}=\left[\Delta u\left(k_{\mathrm{s}} \mid k_{\mathrm{s}}\right), \Delta u\left(k_{\mathrm{s}}+1 \mid k_{\mathrm{s}}\right), \ldots, \Delta u\left(k_{\mathrm{s}}+N_{u} \mid k_{\mathrm{s}}\right)\right]^{\mathrm{T}}
$$

The sum of control increments is obtained as follow

$$
\Delta u_{\mathrm{s}}\left(k_{\mathrm{s}}+i \mid k_{\mathrm{s}}\right)=\sum_{j=0}^{i} \Delta u\left(k_{\mathrm{s}}+j \mid k_{\mathrm{s}}\right)
$$

for $i=0,1,2, \ldots, N_{u}$, which produces the following control sequence

$$
\Delta \boldsymbol{U}_{\mathrm{p}}\left(k_{\mathrm{s}}\right)=\left[\Delta u_{\mathrm{s}}\left(k_{\mathrm{s}} \mid k_{\mathrm{s}}\right), \Delta u_{\mathrm{s}}\left(k_{\mathrm{s}}+1 \mid k_{\mathrm{s}}\right), \ldots, \Delta u_{\mathrm{s}}\left(k_{\mathrm{s}}+N_{u} \mid k_{\mathrm{s}}\right)\right]^{\mathrm{T}}
$$

Compensator Node. The compensator adopts predictive compensation schema as follow

$$
u_{\mathrm{c}}(k)= \begin{cases}u_{\mathrm{c}}\left(k_{\mathrm{s}}^{*}-1\right)+\Delta \boldsymbol{U}_{\mathrm{p}}\left(k-k_{\mathrm{s}}^{*}\right) & k-k_{\mathrm{s}}^{*}<N_{u} \\ u_{\mathrm{c}}\left(k_{\mathrm{s}}^{*}-1\right) & k-k_{\mathrm{s}}^{*} \geq N_{u}\end{cases}
$$

where $u_{\mathrm{c}}(k)$ is the real control value, $k_{\mathrm{s}}^{*}$ is sensor timestamp of the last packet.

\section{Simulation Results}

Consider Hammerstein system described as

$$
\left\{\begin{array}{l}
x(k)=1.5 u(k)-1.5 u(k)^{2}+0.5 u(k)^{3} \\
y(k+1)=0.6 y(k)-0.1 y(k-1)+1.2 x(k)-0.1 x(k-1)
\end{array}\right.
$$

The reference signal is chosen as

$$
r(k)=3+(-1)^{\wedge} \operatorname{ceil}(k /(T / 2+1))
$$

where ceil(arg) is a function that computes the smallest integer value not less than arg.

The controller adopts the MFAPC, PI-MFAPC and DBNPC method respectively. The initial conditions are set to be $u(1: 4)=u_{\mathrm{c}}(1: 4)=0, y(1)=0, y(2)=0.1, y(3)=0.2, y(4)=0.3$; packet loss rate: 20\%; MFAPC: $N=6, N_{u}=4, \eta=1, \mu=1, \hat{\varphi}(1)=1, \varepsilon=10^{-5}, \delta=0.8, \lambda=12$; PI-MFAPC: $k_{\mathrm{P}}=$ 0.38, $k_{\mathrm{I}}=0.5$; DBNPC: $d=20, \eta=1, \mu=1, \varepsilon=10^{-5}, \rho=1, \hat{\varphi}(1)=1, \lambda=6$.

Remark: The packet loss characteristic of the network is described by the Bernoulli distribution.

The simulation result is shown in Fig. 2, which indicates that that the output tracking performance is well achieved by PI-MFAPC method clearly. 


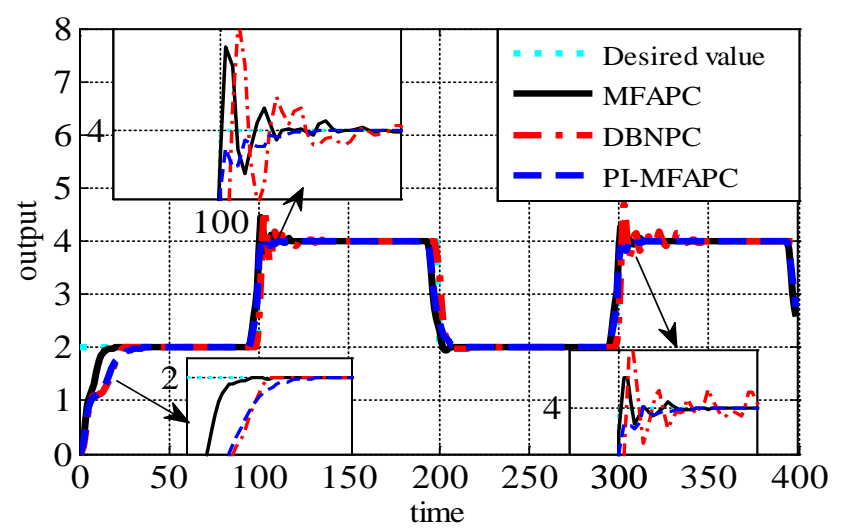

Fig.2 The Output of NCS with Different Control method

\section{Conclusion}

For the problem of NCS packet loss, a networked MFAPC control scheme is proposed. The proposed scheme is based on the data-driven predictive control method. It only uses the I/O data of the plant to design the controller, and does not involve the mathematical model of the plant. Then the complicated modeling problem of NCS is avoided. The simulation results show that the improved MFAPC method is robust in network environment and can suppress system oscillation caused by the frequent change of the expected signal or packet loss. Considering both network induced delay and data packet loss together is the focus of further research.

\section{References}

[1] G.C. Walsh, Y. Hong, L.G. Bushnell. Stability analysis of networked control systems [J]. IEEE Transactions on Control Systems Technology, 2002. 10(3): 438-446.

[2] G.S. Tian, F. Xia, Y.C. Tian. Predictive compensation for variable network delays and packet losses in networked control systems [J]. Computers \& Chemical Engineering, 2012. 39(10): 152-162.

[3] A. Rabello, A. Bhaya. Stability of asynchronous dynamical systems with rate constraints and applications [C]. in Proceedings of the 2002 American Control Conference. 2002.

[4] O.C. Imer, S. Yüksel, T. Başar. Optimal control of LTI systems over unreliable communication links [J]. Automatica, 2006. 42(9): 1429-1439.

[4] X. Tang, B. Ding. Model predictive control of linear systems over networks with data quantizations and packet losses [J]. Automatica, 2013. 49(5): 1333-1339.

[5] Y. Xia, G.P. Liu, M. Fu, et al. Predictive control of networked systems with random delay and data dropout [J]. IET Control Theory \& Applications, 2009. 3(11): 1476-1486.

[6] J.M. Lee, J.H. Lee. Approximate dynamic programming-based approaches for input-output data-driven control of nonlinear processes [J]. Automatica, 2005. 41(7): 1281-1288.

[7] Z.S. Hou, Z. Wang. From model-based control to data-driven control: Survey, classification and perspective [J]. Information Sciences, 2013. 235: 3-35.

[8] Y. Xia, W. Xie, B. Liu, et al. Data-driven predictive control for networked control systems [J]. Information Sciences, 2013. 235(6): 45-54.

[9] Z.H. Pang, G.P. Liu, D. Zhou, et al. Data-based predictive control for networked non-linear systems with two-channel packet dropouts [J]. IET Control Theory \& Applications, 2015. 9(7): 1154-1161.

[10] Z. Hou, Y. Zhu. Controller-Dynamic-Linearization-Based Model Free Adaptive Control for Discrete-Time Nonlinear Systems [J]. IEEE Transactions on Industrial Informatics, 2013. 9(4): 2301-2309. 